\title{
ZEEMAN TOMOGRAPHY OF MAGNETIC WHITE DWARFS: GENERAL METHOD AND APPLICATION TO EF ERIDANI
}

\author{
F. EUCHNER, K. BEUERMANN, K. REINSCH, S. JORDAN AND \\ F.V. HESSMAN \\ Universitäts-Sternwarte Göttingen, Geismarlandstr. 11, \\ D-37083 Göttingen, Germany ${ }^{\dagger}$ \\ AND \\ B.T. GÄNSICKE \\ Dept. of Physics $\&$ Astronomy, University of Southampton, \\ Southampton SO17 1BJ, UK
}

\begin{abstract}
We have developed a new method to derive the magnetic field distribution on the surfaces of rotating magnetic white dwarfs from phaseresolved flux and circular polarization spectra. An optimization code based on an evolutionary strategy is used to fit synthetic Zeeman spectra for a variety of model geometries described in the framework of a truncated multipole expansion. We demonstrate that the code allows the reconstruction of relatively complex fields using noise-added synthetic input spectra. As a first application, we analyze flux and circular polarization spectra of the polar EF Eri in a low state of accretion taken with FORS1 at the ESO VLT.
\end{abstract}

\section{Reconstruction of the field geometry from synthetic spectra}

We use a pre-computed database of 46800 synthetic flux and polarization spectra for field strengths of 1-400 MG and a wide range of effective temperatures to generate synthetic Zeeman spectra, which we subject to an automatic optimization scheme with the aim to recover the parameters describing the original magnetic field configuration. We adopt input field geometries which involve the sum of non-aligned dipole and quadrupole components. We also provide for off-centre shifts of the configuration. By

${ }^{\dagger}$ SJ: now at Institut für Astronomie und Astrophysik, Universität Tübingen, Germany 


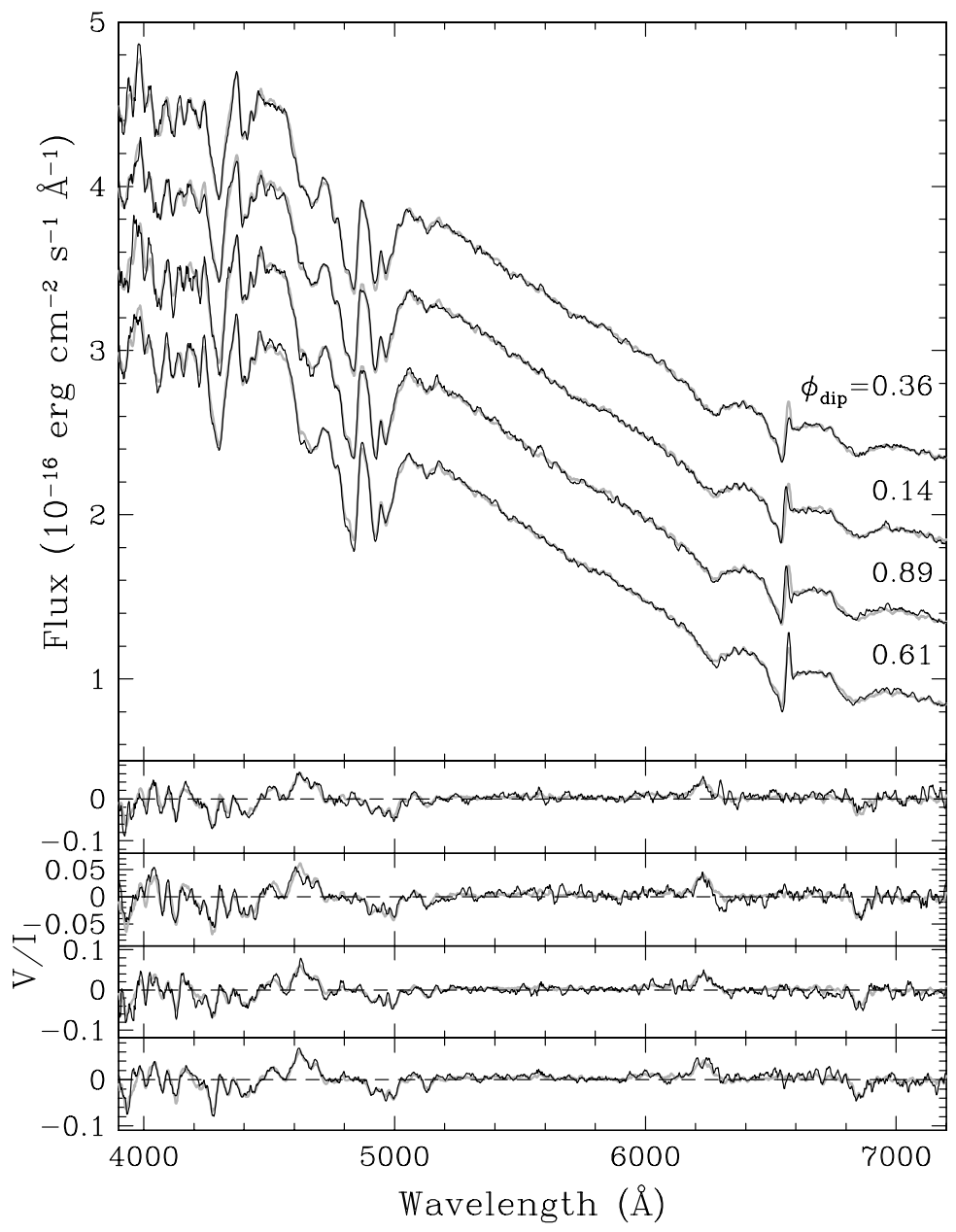

Figure 1. Flux (top) and circular polarization (bottom) spectra of EF Eri, taken on 22 Nov 2000 with FORS1 at the ESO VLT (black: spectrum at phase $\phi$, grey: orbital mean spectrum). The upper three flux spectra have been shifted vertically to avoid overlap.

adding Gaussian noise we create input spectra with $S / N=100$ and 20 , respectively.

Off-centred dipole-quadrupole combinations are reliably recovered even for $S / N=20$ by our code, which can handle up to 12 free parameters but becomes inefficient if higher multipoles are included. The poor convergence is caused by the complexity of the $\chi^{2}$-landscape, which develops an increasing number of secondary minima for more complex field geometries (for a detailed investigation, see Euchner et al., 2002). We find that a given set of phase-resolved Zeeman spectra can be reproduced within the noise 


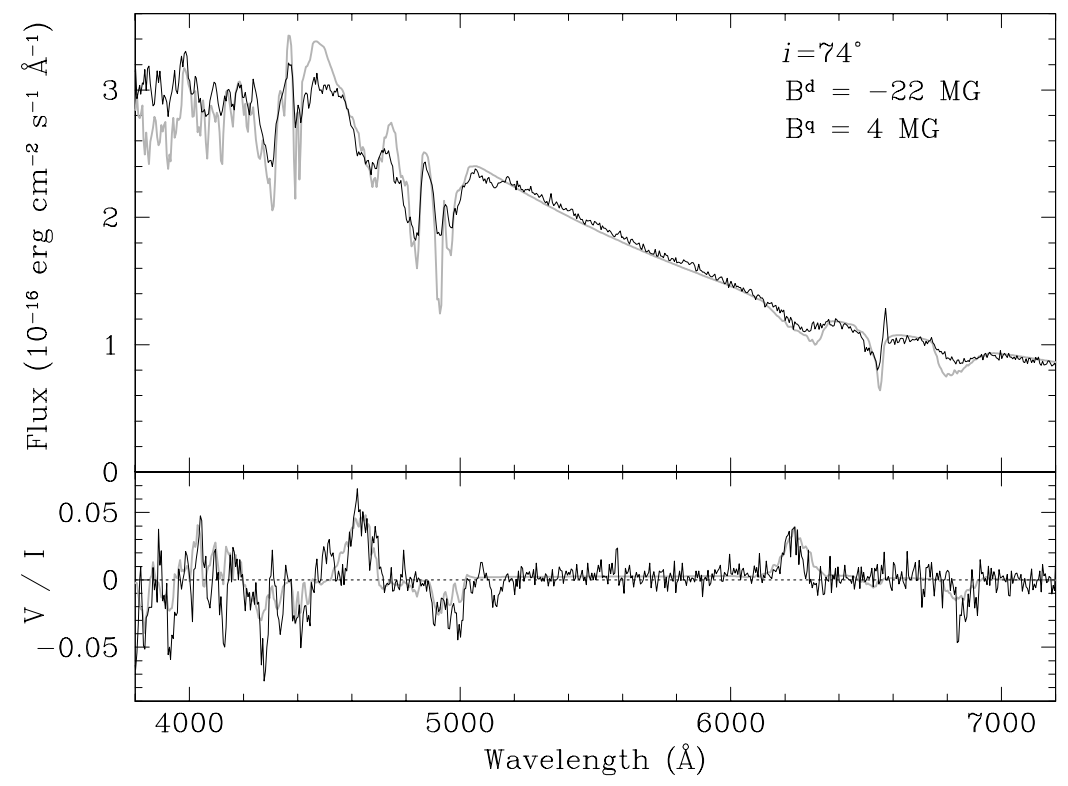

Figure 2. Dipole+quadrupole fit to the orbital mean flux and circular polarization spectra of EF Eri (black: VLT observation from Nov 2000, grey: model).

by quite different formal representations of the field, which, however, all seem to describe rather similar actual field geometries. We are confident, therefore, that observed phase-resolved Zeeman spectra can provide quite definite information on the field structure.

\section{The magnetic field of EF Eridani}

The magnetic field structure over the surface of accreting white dwarfs in cataclysmic binaries becomes accessible to observation only in states of low or switched-off accretion. Following the first detection of Zeeman lines in the polar EF Eridani $\left(P_{\text {orb }}=81 \mathrm{~min}\right)$ by Wheatley \& Ramsay (1998), we obtained phase-resolved flux and circular polarization spectra of this object with FORS1 at the ESO VLT on 22 Nov 2000. Fig. 1 shows the spectra collected in four almost equal phase bins after removal of a slight rotational temperature variation. There is no obvious variation of the Zeeman structures with phase. Fits of inclined multipole field models with the angle $\beta$ between magnetic and rotational axis left free yielded $\beta \simeq 0$. The lack of phase dependence of the Zeeman features further suggests that the tesseral/sectoral multipole components are weak. We fitted the mean flux and polarization spectra, therefore, with an expansion including only the zonal $(m=0)$ components up to a maximum degree $l=l_{\max }$. 


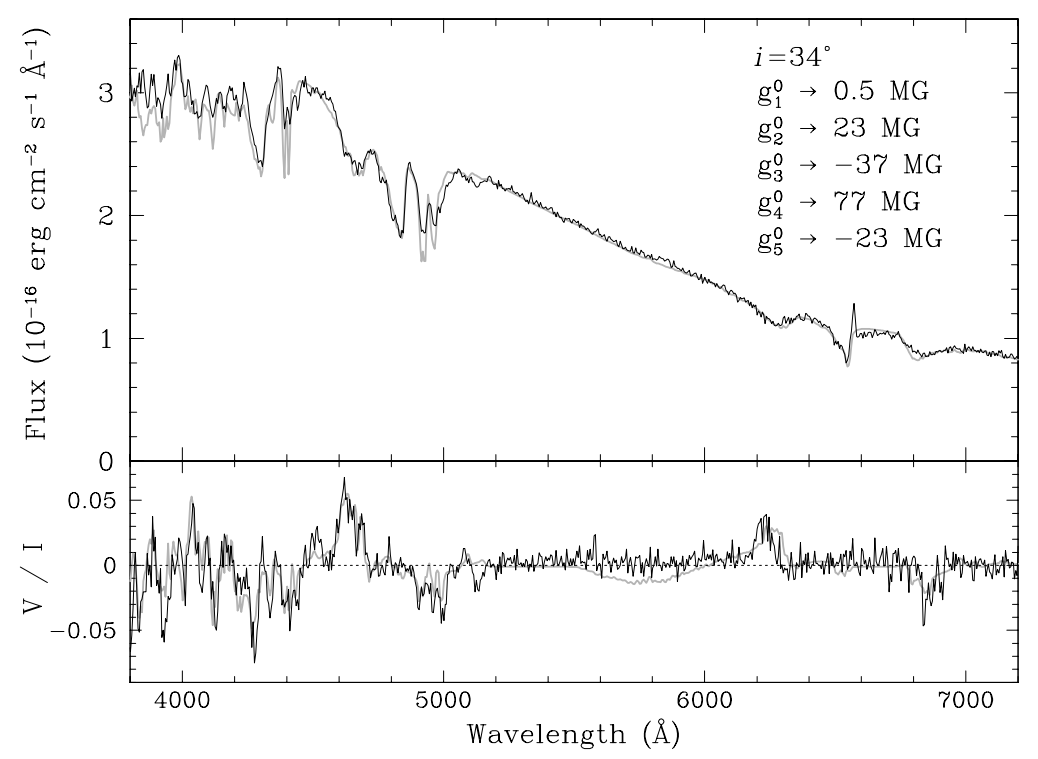

Figure 3. Higher-order multipole fit to the orbital mean flux and circular polarization spectra of EF Eri (black: VLT observation from Nov 2000, grey: model).

Fig. 2 shows the dipole+quadrupole fit $\left(l_{\max }=2\right)$, which is substantially better than the pure dipole (not shown). Fig. 3 depicts the result for the expansion up to $l_{\max }=5$. The dipole is replaced by large coefficients for the higher-order multipole components which substantially improve the fit. While the higher-order multipole fit accounts for many of the observed details - all the wiggles in the blue are genuine Zeeman features - there are still noticeable discrepancies: (i) the observed lack of circular polarization around $5700 \AA$ requires that a larger fraction of the $B \sim 40 \mathrm{MG}$ field occurs at negative $\cos \psi$ (angle between line of sight and field direction), and (ii) the observed narrow spectral feature at $5120 \AA$ can be fitted by additional field contributions with $B$ between 60 and $100 \mathrm{MG}$ pointing away from the observer, possibly generated by a steeper rise of $B$ towards the lower (unseen) pole than provided by the model.

We conclude that the magnetic field of EF Eri deviates strongly from a centred dipole, but do not pretend to have arrived at a definitive solution for its field structure.

\section{References}

Euchner, F., Jordan, S., Beuermann, K., Gänsicke, B. T., \& Hessman, F. V. (2002) AछA 390, 633-647.

Wheatley, P. J. \& Ramsay, G. (1998) in Wild Stars in the Old West, ed. S. Howell, E. Kuulkers, \& C. Woodward, ASP Conf. Ser. No. 137, 446-447. 